\title{
A REVIEW ON SIGNATURE DETECTION AND SIGNATURE BASED DOCUMENT IMAGE RETRIEVAL
}

\author{
Chinnu S Gupta ${ }^{1}$, Umesh .D. Dixit ${ }^{2}$ \\ ${ }^{1}$ M.Tech, E\&CE dept, B.L.D.E.A's College of Engineering and Technology, Vijayapur 586101, India \\ ${ }^{2}$ Asst. Prof in E\&CE dept, B.L.D.E.A's College of Engineering and Technology, Vijayapur 586101, India
}

\begin{abstract}
Transforming a paper document to its electronic version in a form suitable for efficient storage, retrieval, and interpretation continues to be a challenging problem. Signature is an individualistic identification of a person. It is an authentic identification because a signature cannot be copied by others. Signatures are a special case of handwriting subject to intra personal variation and inter personal differences. To counter check fraud and forgery of handwritten signatures, Signature extraction from printed text background and signature based document retrieval from a large dataset is necessary. A lot many techniques have been implemented successfully for both signature extraction and signature based document retrieval. This paper present techniques and methods evolved for signature extraction and signature based document retrieval.
\end{abstract}

Keywords: signature detection, signature extraction, Document image Retrieval, Query image retrieval

\section{INTRODUCTION}

A signature is an individualistic, unique, evidentiary entity. It provides an important form of indexing that enables effective image search and retrieval from large heterogeneous document image collections. In this paper, we surveyed different technique involved in retrieval system that automatically detects, segments, and matches signatures from document image with unconstrained layouts and complex background. This would involve extracting all the signatures from the documents and then performing a match on these signatures. In searching complex documents, a task of Relevance is relating a signature in a given document to the closest matches within a database of document given a database of signed document. The signature based document retrieval has many of the applications and some of them are listed below.

- For business documents.

- Government organizations.

- Digital libraries for online books, student thesis etc.

- Security requirements of document.

The main contributions of this paper are summarized as follows. Firstly, in this paper we have provide general framework and detailed survey of signature extraction and signature based document retrieval. Secondly, we have discussed about issues and challenges.

The paper is organized as follows. In section 2, we given the general framework and in section 3 , we review the related work. The issues and challenges in section 4 , in section 5 , we discuss the performance evaluation metrics. Section 6 , concludes the paper

\section{GENERAL FRAMWORK}

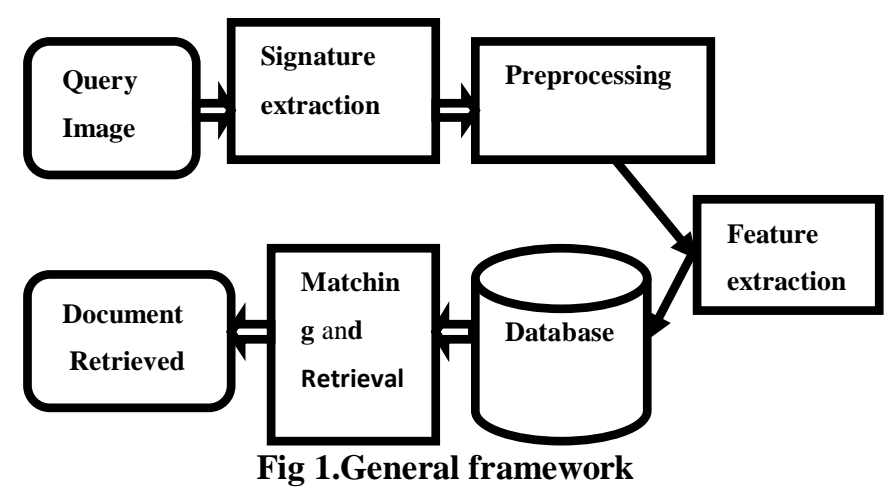

The figure shows general framework for signature based document retrieval.

- Query image: The image containing signature is called as query image.

- Signature extraction: It is the process of extracting only the signature part of the document i.e. separating signature and non-signature part of the document.

- Preprocessing: The preprocessing phase is a sequence of image transformations creating the best possible input for feature extraction algorithms. Preprocessing step may include filtering, RGB to gray scale conversion and binarization.

- Feature extraction: Feature extraction is a set of (usually) independent functions returning a characteristic feature set for the input image. Features can either be particular to the whole signature (global features) or to a part of the signature (topological or local features).

- $\quad$ Matching and Retrieval: Given a few available query signature instances and a large database of document containing signatures, the problem of signature 
matching is to find the most similar signature samples from the database. By constructing the list of best matching signature documents, we effectively retrieve the set of documents authorized or authored by the same person.

\section{RELATED WORK}

Madasu, et al. [1] and Chalechale, et al. [2] used geometric features including area, circularity, aspect ratio, size and position to analyze segmented regions. These features are compared based on certain parameters such as Manhattan distance. The crop method objective is to locate rectangular box around the signature which is the object of interest and remove other objects outside this area, like signatures on bank cheque. The crop method works by moving four vectors from the four different directions (namely up, right, bottom and left) towards the object of interest. Each vector will mark the border of each side of the rectangular box. The crop is then applied to area. Once the area approximation has been done on the first model of the cheque. It is then easy to extract a signature from a bank cheque and scan it. The method failed to extract the signature as the signature was not in the stipulated area hence the system could approximate the area to be cropped.

Djeziri, et al. [3] dealt with the signature extraction problem by an approach that was to mimic the human visual perception. They introduced the filiformity as a criterion for the curvature characteristics of handwritten signatures. Filiformity is defined for two topological measures for binary objects which also includes gray level images. It differentiates the contour lines of the signatures from the handwritten lines which are being isolated. The process of isolating is also used to provide measure for local values regarding the whole image. This process fails when other filiformity objects are present in the document.

Madasu, et al. [4] tried to crop the image segment by estimating the area in which the signature lies using a sliding window. They then analyzed the local entropy derived from the pixel-based density of the region to decide its being signature or not. This approach disregarded the noise and therefore high-density regions are reported as signatures incorrectly. This segmentation does not need any a priori information about the data field and features.

Ritesh Banka, et al. [5] proposed the method of extracting signature from cluttered background which is scale invariant technique using the efficiency of symmetric and loop features that are common in signatures for extracting the standard signatures from other documents. Signature is a handwritten text hence this technique can be used because signatures in English contain most of the characters exhibiting symmetry in vertical, horizontal or diagonal directions. The self printed characters from the documents, as a few papers, lack of proper self symmetry and are misclassified as handwritten text at the first level. The misclassified printed characters in the first level contain a single symmetric loop, which discriminates them from hand written elements. The algorithm had two domains character level and document level. For poor quality document where the numbers of characters joint and broken are very high, the algorithm efficiency decreases.

R.Jayadeyan, et al. [6] considered the images of bank document a method has been proposed for the extraction of signatures, signature localization is done using variance, signature block extraction is done using entropy, normalization are used for desired mean and variance, a hidden Markov model (HMM).Horizontal and vertical projection, right and left envelope, top and bottom envelope, horizontal and vertical size variations of the binary format of the genuine signatures are considered as the feature set of an individual.

Sha'ashua, et al. [7] introduced the concept of multi-scale saliency feature for signature detection that defines signature characteristics by identifying salient structure by grouping contours. They defined a saliency function which increased with the curve length and decreased with the curvature when totally squared. This was done by locally connected network. Their approach was confined to rigid objects having 1-D contour. This can be applied to handwritten notes collected on a tablet pc online since trajectories of the pen are available the framework used is general as it does not embed unique assumption on local feature of the object for example stroke level features for signatures. Hence it is robust against any changes in shape based object detection problems and is applicable to different languages.

A three-stage procedure was proposed by Mandal, et al. [8] to extract signatures. First an algorithm is used to locate the Signature in the document using word-level feature extraction. Second stage separates signature strokes that overlap with the printed text. Final stage uses conditional random field minimization energy concept with skeleton analysis to classify real signature strokes. Improper segmentation of strokes causes error in separating signature from printed text.

Bassam, et al. [9] used the method for extracting signature from image on document is the base proposed auto cropping method. This method improves the performance of security system based on signature image as possible the region of interest of the used image for the biometric system and it also reduces the time cost associated with signature. Auto cropping is the fast procedure to extract the Region of interest (ROI). In this method they used image segmentation and extraction. This cropped signature has no garbage region it crops only the ROI of signature image. This takes less processing time then the original signature image. The performance of this method shows through its speed time, and keeping the content information of the signature object without losing of any pixel of image.

Esteban, et al. [10] considered two main stages of extraction one being comparing distribution of stroke in model signature against distribution of stroke in the original signature using a different approaches extraction being the main concern accumulative evidence technique is used, and 
they achieved a high accuracy with this approach. Their assumption is not usually true since the bank application forms are often filled by new customers and this process is not followed by verification but an archiving step.

Tang, et al. [11] proposed a technique to automatically gain knowledge from various types of documents because knowledge acquisition is difficult to obtain. It was proposed to gain automatic knowledge acquisition in document images by analyzing the geometric structure and logical structural images. These two structures play an important role to process the knowledge acquisition. The limitations of these techniques are to find which is right in transforming a geometric structure into a logical structure. Another difficulty is to find the correct rules involved in obtaining knowledge from different documents.

Liu, et al. [12] presented an approach to image-based form document retrieval and they proposed a similarity measure for forms that is insensitive to translation, scaling, moderate skew, and image quality fluctuations, and developed a prototype form retrieval system based on their proposed similarity measure.

G.Zhu, et al. [13] proposed the method for signature based document retrieval performance using different shape representation like salient contour computed by detection and segmentation of the skeleton that are extracted directly from labeled signature region of database .The thinning algorithm shows sensitivity to structural variations due to noise and neighboring stroke salient contours give a globally consistent representation of structurally important shape feature. This retrieval technique has been successful on the Maryland Arabic dataset in which background handwritten as well as signature are closely spaced.

Chalechale, et al. [14], proposed signature based decomposition and retrieval of document images, and he investigated Arabic/Persian signature recognition and retrieval. For the conduction of retrieval automatic links were considered between feature vector of the signature and the document containing the image using file names. The retrieval performance was measured by average normalized retrieval rate. The ANMRR indicates better performance in comparison with line segmentation distribution method.

A.chalechale, et al. [15] proposed new method for document image decomposition based on connected component analysis and geometric properties of labeled region. The signature is detected and extracted by spatial partitioning by accumulating pixels and by using magnitude and Fourier transform they achieved rotation invariance. The main objective is signature extraction from the original image and converted into a compact feature vector that supports measuring signature similarities.

Srihari, et al. [16], proposed a document image retrieval using signature as queries. Using global shape binary feature vector, a normalized correlation similarity measure for signature matching is done. The objective is to retrieve the closest matching signature obtained from dataset by the same signature person to remove the printed text from the signature image. An image enhancement procedure using chain code information is used. The technique gave promising result for group based and non grouped base retrieval with accuracy and precision.

H.Srinivasan, et al. [17] used conditional random fields and proposed the method for signature based retrieval. In this they retrieved the document from database using signature image as query. Isolating the different contents present in the document and with the help of CRF in extracting signature from complex document. This method presents the signature retrieval strategy using document indexing and retrieval. Indexing is done by using a model based on CRF. SVM is also supported by this technique, and the CRF is used to label each patch and identified using the labels of the neighboring patches. Document retrieval is performed by using matching algorithm to compare the query with the signature.

Guangya Zhu, et. Al. [18], proposed a signature-based document image retrieval system that automatically detects, segments, and matches signatures from document images with unconstrained layout and complex backgrounds .Signature is treated as a non rigid shape and represented by discrete set of 2-D point to most commonly sited measures for retrieval R-precision and average precision. The Rprecision emphasizes the ranking among retrieve documents. Extensive experimental and field test give excellent performance for document retrieval

\section{ISSUES AND CHALLENGES}

The issues and challenges for signature extraction and retrieval are listed below.

- Signature with low resolution in documents make difficult for detection and segmentation.

- The background of document differs from each other.

- The computer vision faces an important problem of Detecting, segmenting and matching deformable objects such as signature.

- Documents are subjected to restricted processing time due to urgency of applications. Therefore the detection and retrieval time must be fast.

- The handwritten characters and auxiliary lines contained in the document overlap and resemble signatures.

\section{PERFORMANCES AND EVALUATION}

\section{METRICS}

- Accuracy: It is the percentage of number of correctly detected signatures to the number of groundtruthed signatures from the document image.

$$
\text { Accuracy }=\frac{\text { no. of correctly detected signatures }}{\text { no. of signatures in groundtruth }}
$$


- Precision: This refers to the percentage of retrieval of document images that are relevant to query [19].

$$
\text { Precision }(N)=R_{n} / N
$$

Where $\mathrm{N}$ is number of retrieval and $\mathrm{R}_{\mathrm{n}}$ is number of relevant matches among retrievals.

- Recall: This refers to the percentage of all the relevant document images in the search database which are retrieved.

$$
\mathrm{R}_{\mathrm{e}} \text { call }(\mathrm{N})=\mathrm{R}_{\mathrm{n}} / \mathrm{M}
$$

Where $\mathrm{M}$ is the total number of relevant matches in the database $R_{n}$ is the number of relevant matches among retrievals.

A strategy for evaluating document image retrieval system involves the following techniques. These techniques have been partially successful in signature based document retrieval. The technique involves extraction of signature from certain documents by different methods. Matching of signature is done by several method and retrieval by known successful techniques.

\section{CONCLUSION}

This paper provides detailed survey of techniques, as it overcomes the difficulty and challenges faced during extraction of signatures from the window of different background documents, cheque etc. This paper provides the overview of methods to apply according to the type of the background document and signature window. These newly developed methods are going to give a high rate of recognition. The performance of the most renowned methods provided the higher signature recognition rate with a greater accuracy in extraction and recognition of the signatures and signature based documents. It also highlights issues and challenges in this area.

\section{REFERENCES}

[1]. V K Madasu ,M H M Yusof M Hanmandilu K Kubika,"Automic Extraction Of Signatures From Bank Cheques And Other Documents", DICTA'03.2003.

[2]. A.Chalechale, G.Naghdya, P.Permaratne, A.Martins." Document Image Analysis and Verification using Cursive Signature", IEEE International Conference on Multimedia and Expo, 2004.

[3]. S.Djeziri,F.Nouboud,R.Plamondon, "Extraction Of Signatures From Check Background Based On A Filiformity Criterion", IEEE Trans. Image Process. 7(10), 1425-1438, 1998.

[4]. V.K. Madasu, B.C. Lovell," Automatic Segmentation and Recognition Of Bank Cheque Fields" Digital Image Compute. Tech. Appl., 80(1): 33-40. 2005

[5]. Ritesh Banka, Farshad Nourbakhsh,"Exraction Of Signature \&Handwritten Region From Official Binary Document Image", september, 2008

[6]. R. Jayadevan et al," Variance based extraction and hidden Markov model based verification of signatures present on bank cheques", International Conference on Computation Intelligence and Multimedia Applications. 2007

[7]. G. Zhu, Y. Zheng, D. Doermann, S. Jeager, "Multi-scale Structural Saliency for Signature Detection", IEEE Conference on Computer Vision and Pattern Recognition. 2007

[8]. R. Mandal, P.P. Roy, U.Pal, "Signature Segmentation from Machine Printed Documents using Conditional Random Field", International Conference on Document Analysis and Recognition. 2011.

[9]. BassamAl-Mahadeen and Mokhled S,Islam H.AlTarawneh, "Signature Region of interest using auto cropping", IJCSI,march 2010

[10]. J.L.Esteban, J.F. Vélez, Á. Sánchez, ”Off-Line Handwritten Signature Detection By Analysis Of Evidence Accumulation", IJDAR, 15:359-368. 2012.

[11]. Y.Tang, C.D.Ya, and C.Y.Suen, "Document Processing for Automatic Knowledge Acquisition. IEEE Trans.Knowledge and Data Eng”, vol.6, no.1, pp.3-21,1994 [12]. J. Liu and A.K.Jain"Imaged-Based Form Document Retrieval", Pattern Recognition, vol.33, no.3, pp.503-513, 2000.

[13]. Guangyu Zhu, Yefeg Zheng, and David Doermann,"Signature Based Document Image Retrieval", ECCV, 2008, Part III, LNCS 5304, pp.752-765, 2000.

[14]. Abdullah Chalechale, Golshah Naghdy, "Signature Based Document Retrieval. Faculty Of InformationPapers", University of Wollongong.

[15]. Sargur N. Srihari, Shravya Shetty, Gady Agam and Ophir Frieder .2006. Document Image Retrieval Using Signature as Queries. In Proceedings of the Second International Conference on Document Image Analysis for Libraries (DIAL'06).

[16]. H. Srinivasan and sargur Sridhar," Signature-Based Retrieval Of Scanned Document Using Conditional Random Field",2009.

[17]. Ilkhan cuceloglu, Hasan Ogul,'Detecting Handwritten Signature In Scanned Documents “, February, 2014

[18]. M. B. Kokare and M. S. Shirdhonkar, "Document Image Retrieval: An Overview", International Journal of Computer Applications, vol. 1, no. 7, (2010), pp. 114-119.

\section{BIOGRAPHIES}

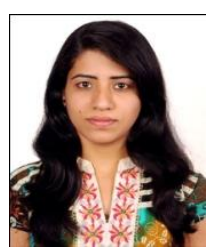

Miss. Chinnu s. Gupta has completed her Bachelor of Engineering in Visvesvaraya Technological University, Belgaum. Currently pursuing Master in Technology, from the same university. Area of interest in Image Processing.

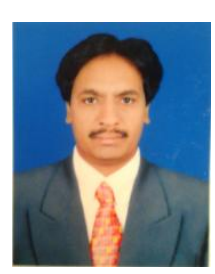

Mr. Umesh .D. Dixit has completed his BE And M.Tech from Visvesvaraya Technological University, Belgaum. He is working as Asst.Prof.in the department of E\&C, BLDEA's CET, Vijayapur, since 11 Years. Currently he is pursuing $\mathrm{PhD}$ in Visvesvaraya Technological University,

Belgaum. 